\title{
Experimental study on the onset of positive corona in atmospheric air
}

\author{
McAllister, lain Wilson; Crichton, George C; Bregnsbo, E.
}

Published in:

Journal of Applied Physics

Link to article, DOI:

10.1063/1.325875

Publication date:

1979

Document Version

Publisher's PDF, also known as Version of record

Link back to DTU Orbit

Citation (APA):

McAllister, I. W., Crichton, G. C., \& Bregnsbo, E. (1979). Experimental study on the onset of positive corona in atmospheric air. Journal of Applied Physics, 50(11), 6797-6805. https://doi.org/10.1063/1.325875

\section{General rights}

Copyright and moral rights for the publications made accessible in the public portal are retained by the authors and/or other copyright owners and it is a condition of accessing publications that users recognise and abide by the legal requirements associated with these rights.

- Users may download and print one copy of any publication from the public portal for the purpose of private study or research.

- You may not further distribute the material or use it for any profit-making activity or commercial gain

- You may freely distribute the URL identifying the publication in the public portal

If you believe that this document breaches copyright please contact us providing details, and we will remove access to the work immediately and investigate your claim. 


\title{
Experimental study on the onset of positive corona in atmospheric air
}

\author{
I. W. McAllister, G. C. Crichton, and E. Bregnsbo \\ Department of Physics, Building 309B, The Technical University, DK-2800 Lyngby, Denmark
}

(Received 30 January 1979; accepted for publication 2 July 1979)

\begin{abstract}
This paper reports the findings of a study into the gas physical processes which lead to the inception of the positive corona discharge in atmospheric air. A multiple avalanche process is observed to be a basic feature, and correlation with the works reported in the literature suggests that at corona onset the physical phenomena are independent of electrode radius of curvature $\rho$ in the range $0.04<\rho(\mathrm{mm})<250$. The various models used to simulate the precorona discharge sequence and to predict corona-onset field-strength values are then examined. It is concluded that the development of a rigorous physical-mathematical model of corona onset must await a full understanding of the avalanche-to-corona transition mechanism. Prior to this, a simple empirical approach is adequate for the prediction of corona-onset field strengths.
\end{abstract}

PACS numbers: $52.80 . \mathrm{Hc}, 51.50 .+v$

\section{INTRODUCTION}

For atmospheric air there are several methods of predicting corona-onset voltages or, to be more correct, the onset field strengths. Some of these methods are purely empiri-

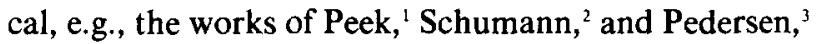
while others are based on considerations of gas physical processes which could conceivably describe the formation of a corona discharge. ${ }^{4,5}$ The values predicted by the different methods are in reasonable agreement. This is not surprising since, with the exception of Peek's formula, each method involves as a dominating factor the function $\exp \left[\int_{0}^{z_{11}} \bar{\alpha}(z) d z\right]$, which describes electron avalanche growth. Consequently, further experimental investigations are necessary in order to determine which of the physical models is the more representative of the conditions existing at corona onset. In this text the term onset applies to the minimum conditions necessary to produce a corona discharge.

In order to study the onset phenomenon it is essential to determine which experimental conditions will initiate a true minimum. Thus, certain parameters such as voltage waveform and electrode geometry must be preselected to ensure that the occurrence of corona at the onset level is readily attained.

A recent study by Bregnsbo et al. ${ }^{6}$ in which a point electrode with a small radius of curvature $(5 \mathrm{~mm})$ was used, has shown that at corona onset the static image of the discharge is comparable to that recorded by other investigators $^{7,8}$ for much larger values of electrode radius of curvature $(\leqslant 250 \mathrm{~mm})$. Hence, the phenomenon of corona onset may be satisfactorily investigated using an electrode with a relatively small radius of curvature. Moreover, as the precorona discharge development, i.e., the formation of electron avalanches, is dependent on the field distribution in the proximity of the point electrode only, a short gap spacing ( $<200$ $\mathrm{mm}$ ) may be utilized. This permits reduction of the necessary insulation clearances to any optical recording system.

As the macroscopic characteristics of positive corona in the onset region are basically identical under impulse and dc voltage conditions, ${ }^{6}$ some of the difficulties encountered in studies with impulse waveforms may be circumvented by using direct voltages. The extremely low probability of the corona event associated with reduced impulse voltage levels can be avoided, and electrical interference in the recording circuits is minimized. This latter aspect is important with respect to the eventual detection and measurement of the precorona discharge activity. In addition, under direct voltages the corona phenomenon can be studied in isolation, whereas under impulse conditions the records obtained (current, charge, light) will normally include aspects of the discharge which should not be directly associated with the onset phenomenon. This distinction is of importance in an examination of the physical details of corona onset.

The present paper reports on the formation and development of the corona discharge in atmospheric air at onset. An attempt is made to elucidate the details of the physical processes active in the discharge. The various corona-onset models are then assessed on the basis of these observations.

\section{EXPERIMENTAL TECHNIQUE}

The high-voltage electrode (cathode) of the experimental gap consisted of an isolated circular horizontal plane (250-mm diameter) with suitably rounded edges. The point electrode (anode) was a 20-mm-diam rod, of overall length $200 \mathrm{~mm}$, with a hemiellipsoidal tip for which the minimum radius of curvature was $5 \mathrm{~mm}$. This rod was mounted vertically on an extensive earth plane such that the gap length was $150 \mathrm{~mm}$. Measurements were made of the gap current, the light emission from the discharge, and the electric field at the point electrode at and below corona onset under the application of a direct voltage.

The gap current was detected by earthing the tip of the point electrode through a coaxial disk resistor $(50 \Omega)$, such that the rise time of the detection circuit was $\sim 1 \mathrm{~ns}$. To record the avalanche current pulses which occur below onset, one stage of preamplification was necessary. This unit had a maximum gain of 100 and a rise time of $\sim 20 \mathrm{~ns}$. Each oscillograph had a bandwidth of $100 \mathrm{MHz}$, corresponding to a rise time of $3.5 \mathrm{~ns}$. A composite oscillogram (see Fig. 6) of the avalanche-to-corona transition could be obtained by utilizing the corona pulse as the trigger signal to the oscillographs. The associated avalanche pulse was electrically delayed, and therefore could be displayed simultaneously with the corona event. 


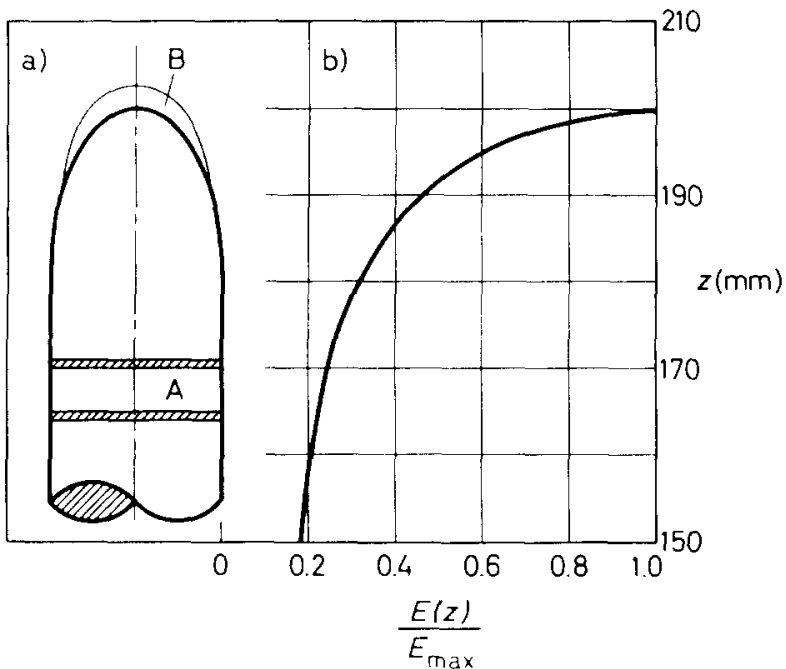

FIG. 1. The point electrode. (a) Location of the field probe (A), and the dimensions of the critical volume (B). (b) The geometric electric field distribution along the electrode surface; $z$ is the axial distance measured from the earth plane.

Since the work of Bazelyan ${ }^{9}$ and Meek and Collins, ${ }^{10}$ many studies have been undertaken in which the effects were recorded of the corona discharge upon the electric field distribution. None of these studies, however, has been associated with a detailed time resolution of the field variations occurring during corona formation. This aspect is examined in the present study.

As the magnitude of the electric field strength changes rapidly along the point electrode profile (Fig. 1), it is advantageous to locate the field probe on the cylindrical shaft where the rate of change is relatively small. Calibration of the field probe was undertaken in a concentric cylinder arrangement.

Light emission from the discharge was recorded by means of several photomultipliers (PM) and an image converter-intensifier system. In the case of the PM viewing the highly stressed region of the gap, quartz optics were used to provide an acceptable degree of spatial selectivity. The increased signal-to-noise ratio obtained permitted electron avalanche activity to be detected at voltage levels well below
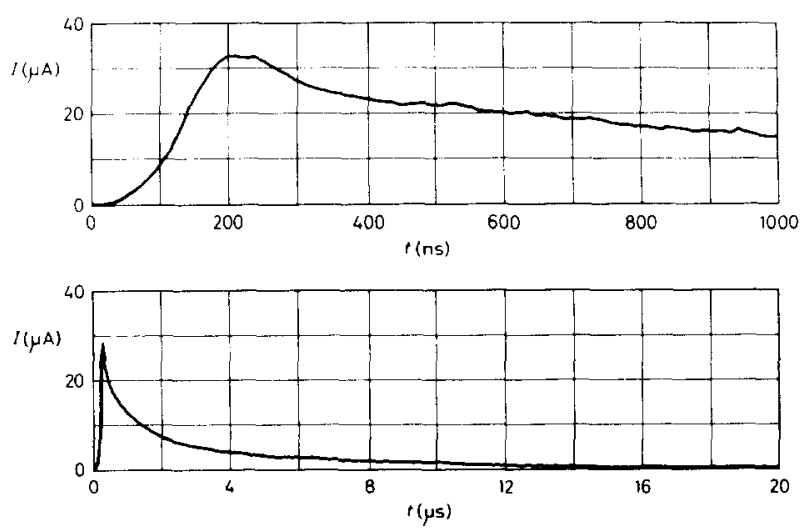

FIG. 2. Electron avalanche current pulses recorded $\sim 1 \%$ below the corona-onset voltage level. corona onset.

The synchronization of the image converter-intensifier recording system with the inception of a corona discharge was achieved by utilizing the associated precorona event (electron avalanche development) as the trigger signal. This event was detected by means of a PM. The triggering system incorporated a nanosecond-trigger amplifier and a pulse generator, such that the inherent overall trigger delay was limited to $\sim 170 \mathrm{~ns}$. This was an essential feature, as previous studies $^{6}$ had shown that the time from avalanche initiation to corona inception was $\sim 200 \mathrm{~ns}$. A storage oscilloscope recorded the PM output signal. From the oscillograms obtained, which displayed either the occurrence or absence of corona, the time-zero of corona formation in each individual case could be established.

\section{EXPERIMENTAL RESULTS}

For ambient air the measured corona-onset voltage was $83.9 \mathrm{kV}$, corresponding to an onset field strength of 5.03 $\mathrm{kV} / \mathrm{mm}$. In the present work the base pressure and temperature are taken as $1 \mathrm{bar}$ and $20^{\circ} \mathrm{C}$, respectively.

\section{A. Below corona onset}

Electron avalanche activity could be detected oscillographically about $2 \%$ below corona onset.

The current records obtained, which are shown in Fig. 2 , illustrate the temporal characteristics of the avalanche pulses; viz., time to the peak, $\sim 200 \mathrm{ns,} \mathrm{and} \mathrm{duration,} \sim 20$ $\mu \mathrm{s}$. These current pulses cannot be analyzed in the manner
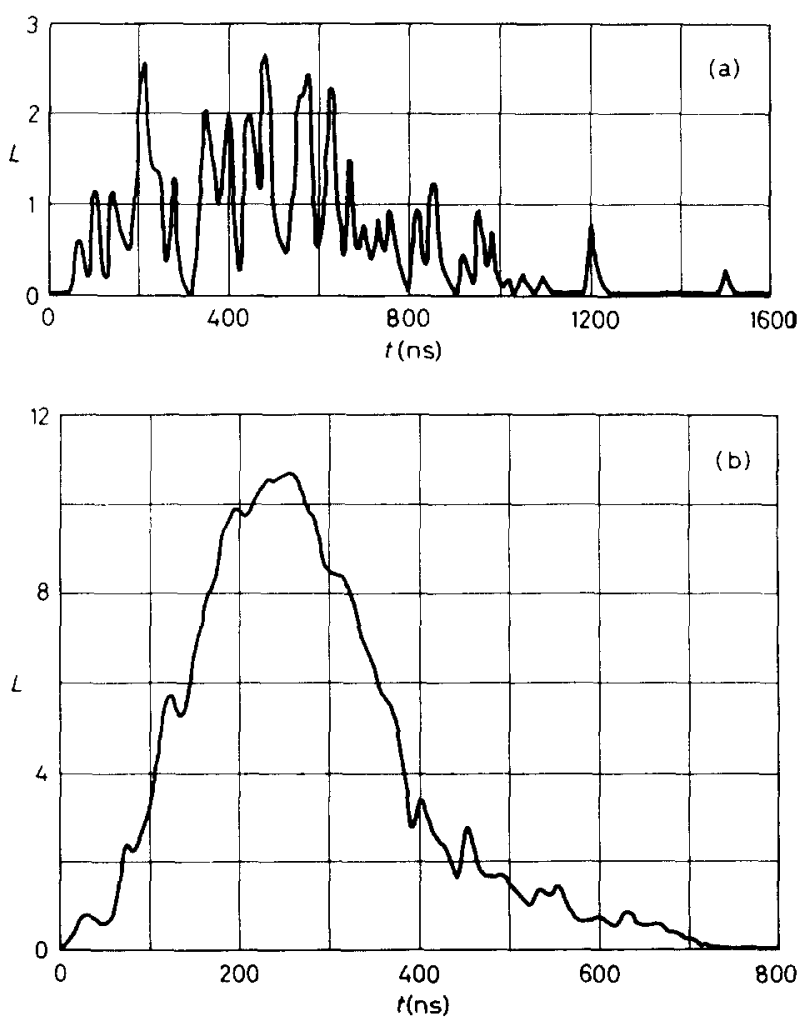

FIG. 3. Photomultiplier records of electron avalanche activity. (a) $\sim 2 \%$ below the corona-onset voltage level. (b) At the corona-onset voltage level. $L$ is the relative light intensity. 
possible under uniform field conditions (see Raether ${ }^{11}$ ) since in this case no simple relationship exists between the elementary discharge data and the form of the current oscillograms. McAllister, ${ }^{12}$ however, has shown that in a divergent field the carrier number associated with an avalanche can be estimated from current pulse measurements. As interest in the carrier number is centered particularly on the conditions existing at corona onset, this aspect will be treated more fully in Sec. III B.

The PM records, shown in Fig. 3, indicate that the ionization activity observed below onset is comprised of a chain of individual electron avalanches with an average generation time of some $40 \mathrm{~ns}$. This short generation time affirms that the cathode of the electrode system cannot play an active role during this stage of the discharge development. Consequently, gas-ionizing radiation is considered to be the mechanism responsible for this generation process.

As the voltage is raised toward the onset level, the records obtained [Fig. 3(b)] indicate that the individual avalanches increase in magnitude such that in each chain a partial overlap of the pulses occurs. The growth of the avalanche chain peaks after $\sim 200 \mathrm{~ns}$ and displays a total time duration of $\sim 700 \mathrm{~ns}$. Due to the limitations imposed by the detection circuit time constant, the individual avalanches could not be satisfactorily resolved in the oscillograms of the gap current. A similar limitation is to be found in the studies of Amin. ${ }^{13}$ However, as a result of the high resolution displayed by the associated light records obtained in the present study, it is evident that the "initial pulse of the burst pulse" (see Ref. 13) is itself a multiple event.

The average change in the field strength across the probe surface, recorded as a function of time, is shown in the oscillograms of Fig. 4. These typical records illustrate that a relatively slow change occurs in the magnitude of the field strength such that the maximum decrease is not observed until after $\sim 25 \mu \mathrm{s}$; the duration of the associated ionization/excitation activity is confined to the first $1 \mu \mathrm{s}$.

After this period of $\sim 1 \mu \mathrm{s}$, all free electrons produced in the avalanche process will have been removed from the discharge volume by the action of the electric field. Subsequent field changes must therefore be associated with the motion of ionic charges. As the applied field strength at the electrode tip is $\sim 5 \mathrm{kV} / \mathrm{mm}$, the ratio of positive to negative ions in the head of the avalanche will be of the order $10^{3}: 1$.
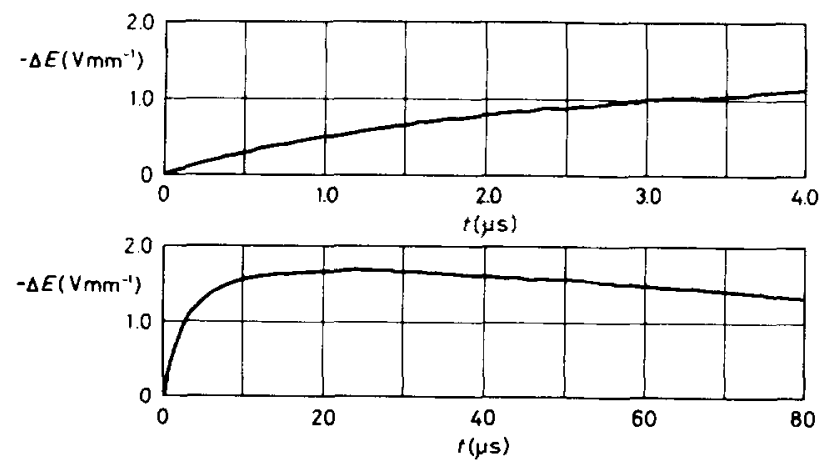

FIG. 4. Decrease in the electric field strength $(-\Delta E)$ due to avalanche development at the corona-onset voltage level.
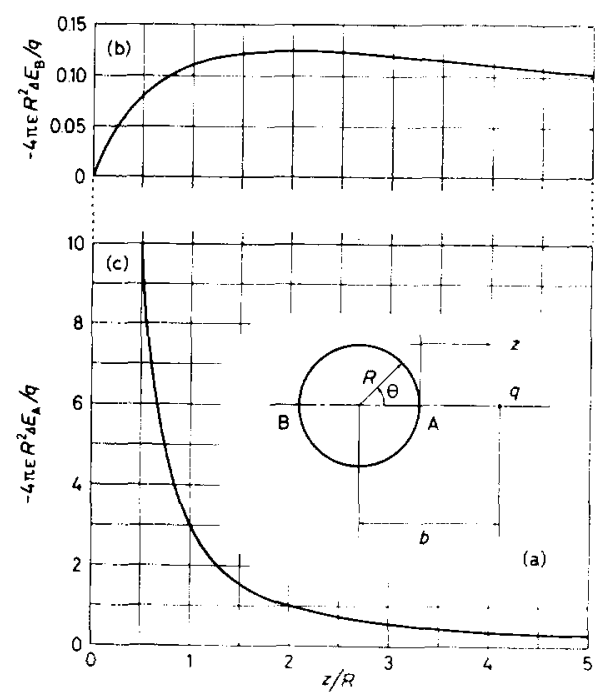

FIG. 5. Simulation of the changes in the electric field produced by the drift of the positive-ion space charge. (a) The analog. (b) Variation of the field change at $B$. (c) Variation of the field change at $A$.

Thus, the maximum field change recorded after $\sim 25 \mu \mathrm{s}$ is considered to be associated with positive-ion motion in the axial field direction. The following analogy, for which a simple analytical solution exists, supports this viewpoint.

As the bulk of the positive ions will be located initially close to the point electrode, the resulting space charge may be represented by an equivalent point charge. The electrode geometry is simulated by an isolated sphere held at a constant positive potential, see Fig. 5 . The change in the field strength at the electrode surface as a function of the position of the point charge can then be evaluated. By considering a relevant positive-ion drift velocity the resulting field changes may be time scaled.

Using the method of images, the field changes produced at the sphere surface by the point charge $q$ can be shown to be given by

$$
\Delta E(\theta)=-\frac{q}{4 \pi \epsilon R}\left(\frac{b^{2}-R^{2}}{\left(b^{2}+R^{2}-2 b R \cos \theta\right)^{3 / 2}}\right) .
$$

Let point $B$ on the sphere surface, $\theta=\pi$, represent the probe position, and point $\mathrm{A}, \theta=0$, represent the electrode tip. In Fig. 5, the variations of the change in the electric field at $\mathrm{A}\left(\Delta E_{\mathrm{A}}\right)$ and $\mathrm{B}\left(\Delta E_{\mathrm{B}}\right)$ are illustrated as functions of the normalized distance from the sphere $(z / R)$. At $\mathrm{B}$ the magnitude of the field change has a maximum value for $z / R=2$; i.e., the maximum change is attained only after the space charge has drifted a specific distance from the electrode, cf. Fig. 4.

At $\mathbf{A}$, however, the magnitude of the field change is a continuously decreasing function. Moreover, when the maximum value of $\Delta E_{\mathrm{B}}$ is attained, the corresponding value of $\Delta E_{\mathrm{A}}$ is still greater by approximately one order of magnitude.

From the experimental results, the maximum reduction in the field at the probe surface is $2 \mathrm{~V} / \mathrm{mm}$ after $\sim 25 \mu \mathrm{s}$. Consequently, to a first approximation, the corresponding field reduction at the apex of the point is $\sim 20 \mathrm{~V} / \mathrm{mm}$. This small reduction ( $\sim 0.5 \%$ of $E_{\text {applied }}$ ) is still of sufficient magnitude to inhibit further vigorous avalanche formation, and 
the minimum time delay observed between chains $(\sim 40 \mu \mathrm{s})$ can be attributed to the fractional suppression of the field strength at the electrode tip. However, the statistics of the inherent production of free charge must play a measurable role in this time lag effect, as delays of up to several hundreds of microseconds are observed.

The overall gain of the combined image converter-intensifier system was not sufficient to allow detection of the light emitted during the avalanche activity. However, by utilizing the intensifier alone, the approximate dimensions of the active volume (i.e., the volume around the point within which ionization/excitation phenomena can occur) could be obtained from integrated records of the avalanche activity $\left(\sim 10^{5}\right.$ single events) at the point electrode. ${ }^{6}$ To emphasize the relative magnitude of the active volume dimensions, the theoretical boundary of this volume is shown in Fig. 1. The limiting field strength of atmospheric air was taken to be $2.42 \mathrm{kV} / \mathrm{mm} .{ }^{14}$

Although avalanche development can occur throughout the active volume, the greatest development will be in the vicinity of the maximum field strength. Thus, the resulting spatial distribution of photoelectrons and the additive effect of the initial space-charge field will induce a strong self-focusing of the subsequent discharge growth. In addition, owing to the extremely low value of the relative charge density, i.e. the ratio of positive ions to neutral molecules in the head of the initial avalanche $\left(<1: 10^{6}\right)$, it is suggested that the subsequent avalanche space charges will fully overlap the primary space charge. Avalanche development will thus occur in a field which is being continuously augmented by space charge until the generation process either terminates, owing to avalanche statistics, ${ }^{11}$ or becomes unstable, precipitating a corona discharge.

The experimental evidence presented in this section clearly illustrates that, at onset, the corona discharge does not result from the formation of a single critical avalanche but from the accumulative effects of an intrinsic multiple avalanche process.

\section{B. Corona-onset region}

When the onset field strength is reached, the occurrence of a corona discharge is most evident, e.g., a distinctive

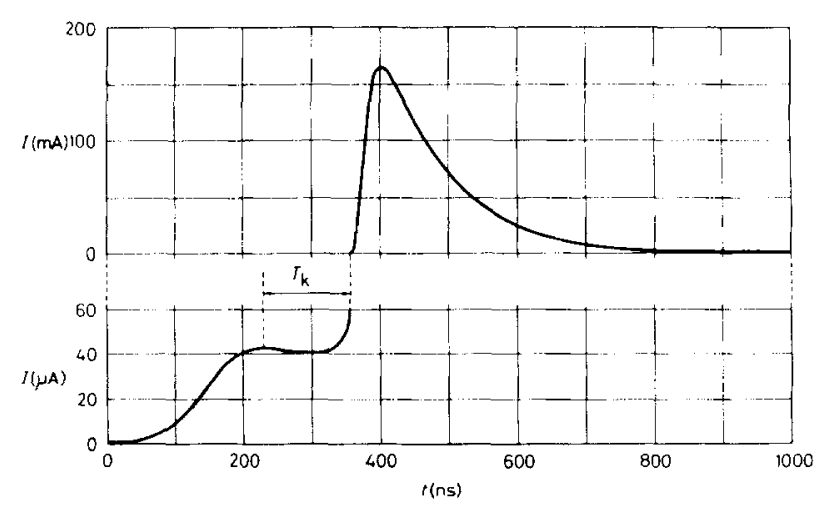

FIG. 6. The avalanche-to-corona transition as indicated by the discharge current. break occurs in the trace of the avalanche current oscillogram. This break is associated with a three orders of magnitude increase in the gap current, which results in the formation of the familiar corona current pulse, see Fig. 6 . The corona pulse displays a time-to-peak of $40 \mathrm{~ns}$ with an apparent duration of some $500 \mathrm{~ns}$. In effect, a transient partial breakdown of the gap occurs.

The avalanche current-pulse oscillograms illustrate that, in order to initiate the corona event, it is first necessary to attain a critical charge level. This critical level may be conveniently related to the peak value of each current pulse. At onset these peak values are consistent to within $3 \%$ and permit a well-defined minimum condition to be established. Consequently, the above observations suggest that a critical avalanche carrier number ${ }^{11}$ is required in order to initiate the corona event, although the concept of a critical-charge density would have more physical relevance.

In the time interval up to this current peak, the recorded pulses will be due predominantly to electron motion since the drift velocity of the avalanche electrons greatly exceeds that of the associated ions ( $>100: 1)$. As mentioned previously, for highly divergent field geometries an evaluation of the electron component of the avalanche current pulse in terms of the carrier number is possible. A reliable estimate of this parameter, however, cannot be undertaken before the response of the detection circuit has been examined. An estimate of the transit time associated with the electron drift indicates that this time is comparable to the time constant of the recording system, and, consequently, the recorded current pulses are subject to a degree of integration.

An analysis of these records, taking into account the integrating effect of the detection circuit, indicated that at corona onset the carrier number associated with the accumulation of the space charge is $\sim 4 \times 10^{7}$. This critical value which describes the minimum condition for corona onset in ambient air compares favorably with the often quoted figure of $\sim 10^{8}$ associated with uniform-field breakdown studies. ${ }^{11}$

The general form of the avalanche pulses leading to corona formation is similar to that recorded in uniform-field studies. "' Each pulse displays a characteristic time-to-gocritical, $T_{k}$; see Fig. 6. $T_{k}$ defines the time interval measured from the initial current peak to the point on the oscillogram at which the rapid increase occurs. At the onset level, $T_{k}$ varies in the interval 100 to $400 \mathrm{~ns}$, but reduces to $\ll 100 \mathrm{~ns}$ for a small increase in the applied voltage, $<0.5 \%$. At onset, as the current peak values are subject to only a small variation ( $3 \%$ ), the relatively large scatter in $T_{k}$ could be associated with the statistics of the reproduction processes e.g., in the production of the free electrons which generate the later stages of the avalanche chain. The actual avalanche-to-corona transition mechanism, however, remains unclear.

As corona formation is associated with a dramatic increase in the level of the light emission from the discharge processes, it becomes possible to record the corona development from its time of inception with the image converterintensifier system. Typical spatial-temporal (streak) records illustrating the major features of the discharge are shown in Figs. 7 and 8 . With reference to Fig. 7(a), it is observed that at onset the corona discharge traverses the entire gap. The 
(a)

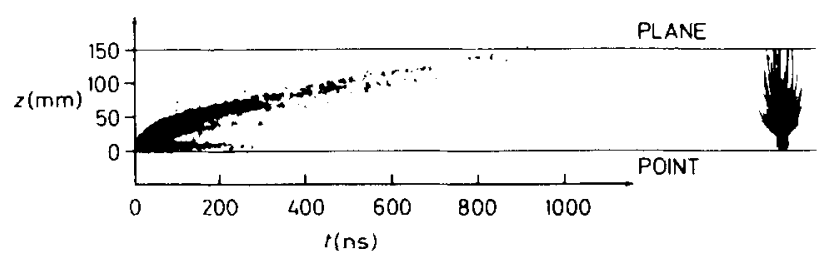

(b)

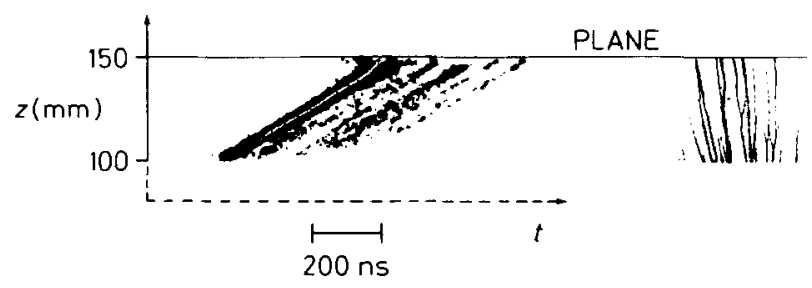

FIG. 7. Spatial-temporal records of corona development. (a) Complete gap. (b) At the plane.

initial section of the discharge, when observed as a narrow channel located on the gap axis, is generally termed the corona stem. The latter section, which is seen to traverse $90 \%$ of the gap terminating on the plane electrode, will be referred to as the corona filament stage.

The corona filaments, which emanate from the stem termination, diverge rapidly from this narrow axial channel as they propagate toward the plane electrode. The filament velocity is estimated to be $\sim 300 \mathrm{~mm} / \mu$ s initially, decreasing finally to $100 \mathrm{~mm} / \mu \mathrm{s}$, such that the plane electrode is reached after some $800 \mathrm{~ns}$.

In Fig. 7(b), owing to the small depth of focus of the converter objective lens compared to the spatial distribution of the filaments, only a few of the filaments are sharply focused. However, their appearance is consistent with the proposal of Park and Cones ${ }^{16}$ in that a filament could be viewed as a small ball of light, indicative of a volume of high ionization and excitation activity, propagating across the gap in the field direction. This proposal has been incorporated by Dawson and Winn ${ }^{17}$ and Gallimberti ${ }^{18}$ in their streamer propagation models.

The corona development in the vicinity of the point electrode is shown in Fig. 8. The typical time-integrated record [Fig. 8(a)] indicates an intense axial development $(\sim 6$ $\mathrm{mm}$ long), from which the filaments evolve. The designation corona stem has been applied previously to similar integrated records. ${ }^{1.8}$ With time resolution, however, the stem is readily observed to be comprised of two components, see Fig. 8(b); viz.,

(1) a single fast phase which propagates with a linear velocity of $\sim 500 \mathrm{~mm} / \mu \mathrm{s}$ for $\sim 10 \mathrm{~ns}$, after which multiple filament development occurs at a much slower rate, and

(2) a glow phase which develops initially at $\sim 150$ $\mathrm{mm} / \mu \mathrm{s}$ and attains its maximum extent from the point electrode after $\sim 40 \mathrm{~ns}$. These records corroborate the description of the corona event given by Dawson ${ }^{19}$ in whose work the terms primary and secondary streamer are used to denote the above events.

The glow phase or secondary streamer is shown in greater detail in Fig. 8(c), from which an apparent duration of $\sim 250 \mathrm{~ns}$ is indicated. In Fig. 8 (c) the time distortion introduced by the off-axis filament development is in evidence, i.e., as the velocity of the filament development is greater than that of the streak velocity these off-axis filaments apparently develop in negative time.

The present records of the corona discharge at onset are identical in their form to the initial stages of the impulse coronas shown by $\mathrm{Dale}^{20}$ and Pesavento $^{21}$ for large-diameter spheres. In the present study the stem structure is clearly viewed to be a two-component event. A similar interpretation is not shared by Pesavento, ${ }^{21}$ who, after referring to a still photograph to classify the stem, apparently equates the stem to the glow phase alone.

Recent spectroscopic studies ${ }^{22,23}$ of the corona discharge have indicated that the glow phase constitutes a region of high electrical conductivity. This aspect should therefore be reflected in the amplitude/temporal characteristics of the corona current, and such a correlation is found to exist in the present study, see Figs. 6 and 8. This observation differs from that of the Les Renardières studies ${ }^{24}$ in which the peak of the current pulse is related in time to the branching point of the primary streamer (i.e., filament inception), and not, as observed in the present study, to the time at which the glow phase attains its maximum axial extent. In the Les Renardières studies a considerable overvoltage is involved, $\sim 30 \%$. In contrast, Gallimberti ${ }^{18}$ associates the measured corona current pulse with filament development, an approach which does not seem compatible with the experimental observations.

A unique feature of the corona discharge at onset is the presence of a single stem. This feature may be explained by assuming that a superposition of the incoming avalanches (space charge) is still required in order to sustain the critical field conditions necessary for the discharge to propagate. When this critical condition can be established by independent groups of the incoming avalanches, branching becomes possible, and the discharge passes into the filamentary stage. Based on the propagation concepts of Dawson and Winn, ${ }^{17}$ the difference in the stem and filament diameter should indicate the diminishing importance of such space-charge superposition in the latter phenomenon. This dimensional differ-
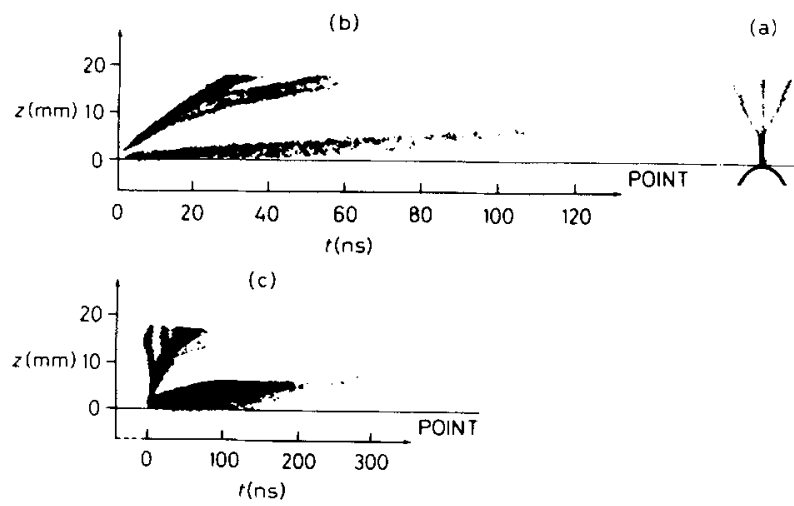

FIG. 8. Spatial-temporal records of the corona development at the point electrode. 
ence tends further to support the concept of charge density rather than that of number.

In order to achieve this transition of the relatively broad stem into a series of narrow filaments, the field stress at the tip of the propagating stem must increase in magnitude as the distance from the electrode surface increases. A function of the highly conducting glow phase may be the establishment of these field conditions; viz., that this phase of the discharge transforms the track of the primary streamer into a semiconducting protrusion, such that the necessary field strengths are realized. Consistent with this interpretation are the observations ${ }^{8.20}$ that the stem length is greater the larger the electrode radius of curvature, and that the stem length decreases with increasing overvoltage.

The PM records of the corona discharge are shown in Fig. 9; (a) relates to the complete gap and (b) to the point region only. Owing to the degree of spatial discrimination introduced, a distinctive difference is to be observed, although a similar maximum light emission is indicated after $\sim 100 \mathrm{~ns}$. The relatively greater activity on the tail of the complete gap pulse is associated with filament propagation across the gap, where arrival at the plane is indicated in the time interval $750-850 \mathrm{~ns}$.

Further confirmation of the time taken by the filaments to traverse the gap was obtained from synchronized PM observations at the plane. The necessary spatial discrimination was introduced by additional slit optics scanning $1-\mathrm{mm}$ sections of the gap. Owing to the spread in the arrival time of the individual filaments at the plane ( $\sim 100 \mathrm{~ns})$ and the resulting excitation produced, an investigation into the possibility of a return phenomenon (e.g., see Ikuta et al. ${ }^{25}$ ) was not feasible. In mid gap, however, where sufficient time discrimination was possible, the only optical activity that could be observed
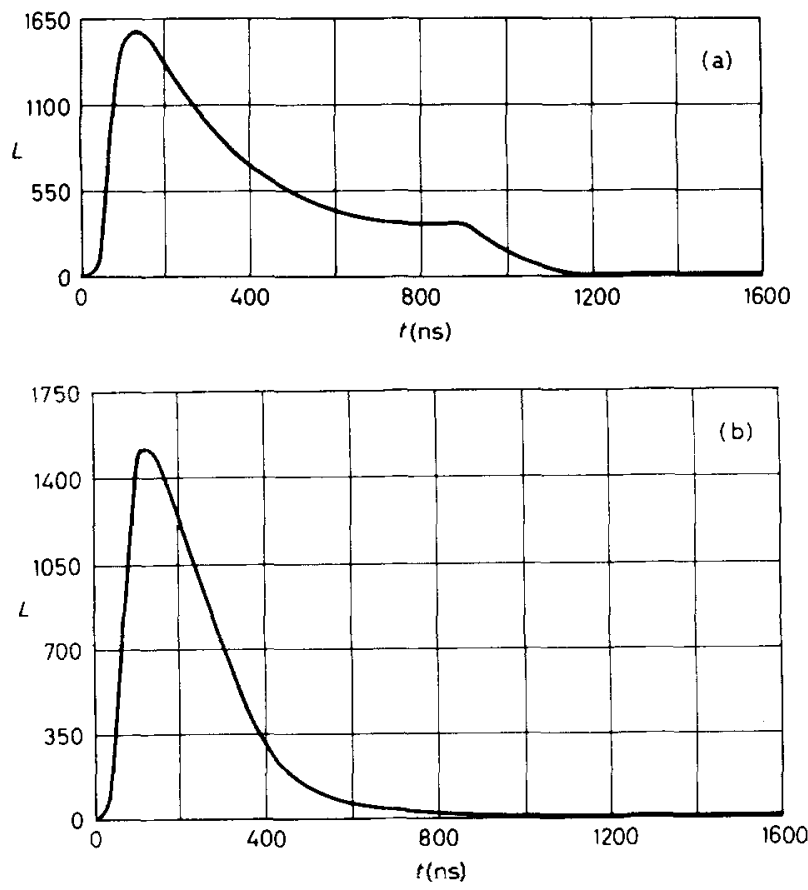

FIG. 9. Photomultiplier records of the corona development. (a) Complete gap. (b) Point region alone. $L$ is the relative light intensity.
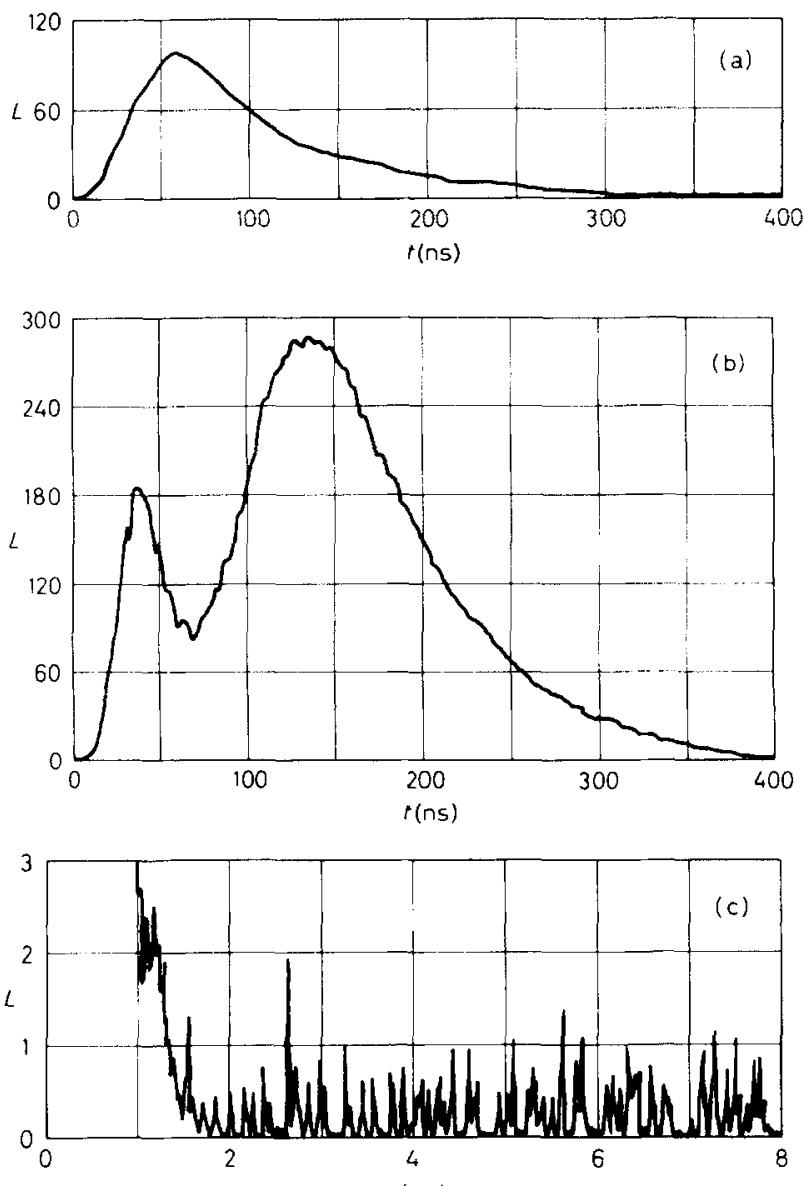

$r(\mu s)$

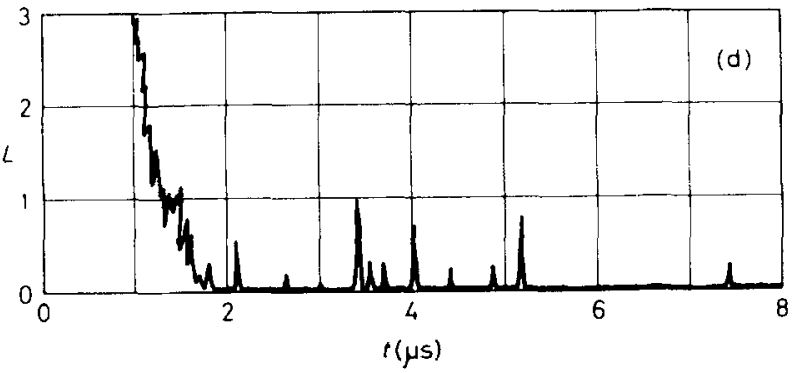

FIG. 10. Photomultiplier records of the stem region obtained using slit optics. (a) and (c) At the electrode tip. (b) and (d) At $5 \mathrm{~mm}$ from the electrode tip. $L$ is the relative light intensity.

was due to the passage of the filament tips toward the plane, a fact compatible with the constant filament velocity observed in the plane region. In the work of Dawson, ${ }^{19}$ a return phenomenon is in evidence but is associated in this case with acceleration of the filament tips as these approach the plane electrode. Thus, it appears that a minimum degree of interaction of the filaments with the electrode surface is necessary in order to initiate a return phenomenon.

With this increase in spatial resolution, the modified PM system was able to corroborate the image converterintensifier recordings; viz., that at the point electrode tip the discharge development is associated with two distinct phases, see Fig. 10. These observations are similar to those made by Ikuta et al..$^{25}$ in gaps with point electrodes of $0.3 \mathrm{~mm}$ radius of curvature.

The investigation of the stem region was extended by 


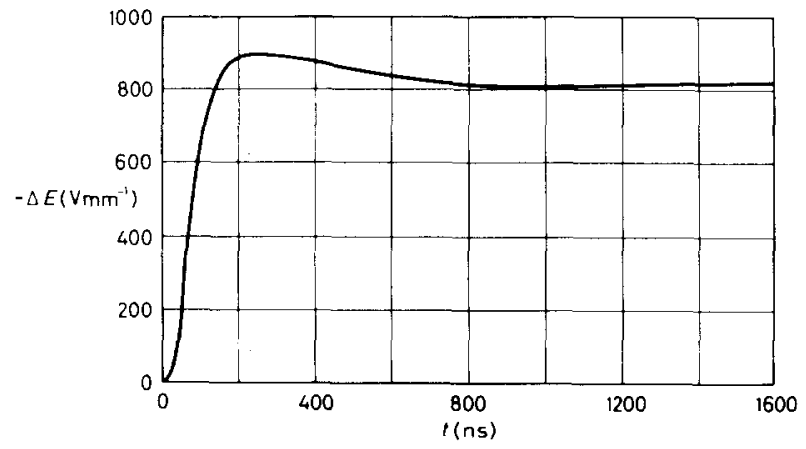

FIG. 11. Decrease in the electric field strength due to corona development.

operating the PM at maximum gain. Under such conditions, the PM behaved in a very nonlinear manner with respect to the corona signal. This mode of operation resulted in the detection of a very low level of light activity for times in excess of $\sim 500$ ns following the decay of the initial intense phenomena. This activity, which was of an intermittent nature, could be detected along the length of the apparently defunct stem for times up to $\sim 2 \mathrm{~ms}$, although in the vicinity of the stem/electrode interface the emitted light was of a more continuous nature, see Figs. 10(c) and 10(d).

A typical field probe record obtained during the corona development is shown in Fig. 11. A comparison with the similar avalanche records (see Fig. 4) illustrates that corona development suppresses the field strength at the point electrode to a much greater extent, $\sim 50: 1$. In such records the arrival of the filaments at the plane, whereupon charge producing activities cease, is clearly indicated by a discontinuity in the trace after $\sim 800$ ns. The ability of the field probe to detect the arrival of the filaments at the plane indicates that the probe is sensitive to the propagation of the filaments across the gap.

As the filaments traverse the gap, the avalanche electrons associated with the active region of each filament will become attached in the corresponding passive region ${ }^{18}$ to provide a negative-ion population. This will counteract the positive-ion population of the previous active region. Thus, with the exception of the newly formed active regions, the filaments should contain comparable positive- and negativeion populations along their paths of propagation. Hence, to a first approximation, the probe response reflects the effective propagation across the gap of a thin positive space-charge layer.

Additional support for this interpretation is the correspondence between the time to the maximum of the probe response $(\sim 250 \mathrm{~ns})$, and the location of the filament tips (layer) in the gap at this time $(t \sim 250 \mathrm{~ns}, z \sim 70 \mathrm{~mm})$. Beyond this location, the number of filaments which continue to propagate steadily reduces. The contour of the discharge, as shown in Fig. 7(a), illustrates this reduction.

For times in excess of $800 \mathrm{~ns}$, the field probe records indicate that a net positive space charge remains in the gap, such that on a $\mu$ s time scale the field reduction at the probe location is approximately constant. This remnant space charge can be associated with the active regions of those filaments which did not traverse the gap. However, as the exact positive- and negative-charge distributions are unknown, it is not possible to relate the probe records to the field conditions at the electrode tip; e.g., a second corona discharge could occur after some $80 \mu$ s even though a considerable field reduction was still in evidence at the probe surface. This second pulse indicates that, owing probably to localized charge separation, the onset field conditions had been reestablished in the tip region. Consequently, the weak light emission observed in this location for $t>500 \mathrm{~ns}$ (see Fig. 10) is probably associated with ionization activity which subsequently results in avalanche formation.

If the assumption is made that such collision phenomena were present prior to this time, and that a high channel conductivity exists, then the stem region of the corona discharge appears to reflect characteristics typical of the positive column in a glow discharge. A hypothesis of this nature has been advanced by Marode ${ }^{22,26}$ in which the overall characteristics of corona are paralleled with those of the glow discharge. In that study, more convincing evidence was available from the detailed observations made on the cathode region of the discharge. The apparent similarity exhibited by these discharge forms has been briefly mentioned by von Engel. ${ }^{27}$

\section{DISCUSSION}

From the long-gap impulse studies of Brago, ${ }^{28} \mathrm{Dale},{ }^{20}$ and Pesavento, ${ }^{21}$ the streak records obtained of the overvolted corona development clearly indicate the presence of a two component stem. In the present work, a similar discharge structure is observed under direct voltages at onset. Hence, it seems reasonable to infer that the same gas physical processes exist at corona onset in gap geometries for which the radii of curvature of the stressed electrodes lie in the range 5 to $250 \mathrm{~mm}$.

In the short-gap regime, the PM records of corona formation presented by, among others, Hudson, ${ }^{29}$ Dawson, ${ }^{19}$ Ikuta et al., ${ }^{25}$ and Korge et al. ${ }^{30}$ are similar in their composition to those obtained in the present work. Consequently, it may be concluded that, at the onset level, corona formation is basically independent of the electrode radius of curvature in the range 0.3 to $250 \mathrm{~mm}$. This lower limit may be extended to $0.04 \mathrm{~mm}$ by including the work of Marode, ${ }^{22}$ although the structural composition of the stem cannot be readily identified from that study. The work of Dawson, ${ }^{19}$ however, indicates that the subsequent stages of these corona discharges are comparable, and an extrapolation to this lower limit of $0.04 \mathrm{~mm}$ seems defensible.

On the basis of this similarity, it appears justified to conclude that within this range of electrode radius of curvature $(0.04<\rho(\mathrm{mm})<250)$, the onset of corona is precipitated by the same basic gas ionizing processes. Thus, with reference to the detailed observations of the corona-onset conditions made in the present work, a critical assessment of the various models can be undertaken.

The majority of the corona-onset models to be found in the literature ${ }^{31-34}$ are based on the assumption of a single electron avalanche developing to some critical condition. These critical conditions range from the simple attainment of an arbitrarily specified carrier number in the electron ava- 
lanche ${ }^{31}$ to considerations of the gain in energy acquired by the electrons during avalanche formation. ${ }^{34} \mathrm{~A}$ well-defined onset condition, which may be associated with a critical carrier number, has been observed in the present study. However, it should be emphasized that this critical number is associated with a multiple avalanche process and cannot be related to the growth of a single avalanche without reservation. In the latter situation, in order to generate this critical number, the application of a somewhat higher field strength would be required owing to the absence of space-charge accumulation.

Numerical estimations ${ }^{17}$ of the magnitudes of the parameters assumed to be essential for the advance of the corona discharge have indicated that, inter alia, a net positive space charge of $\sim 10^{8}$ ions is required. The present measurements confirm that at onset this order of magnitude is not unrealistic. However, the evaluation ${ }^{4}$ of the growth parameter $\exp \left[\int_{0}^{z_{n}} \bar{\alpha}(z) d z\right]$ leads to a value close to $10^{5}$. To account for this discrepancy, Dawson and Winn ${ }^{17}$ calculated avalanche growth based on the assumed continuous buildup from several photoelectrons acting within the critical volume, such that, if aided by space-charge field enhancement, the value of $10^{8}$ could be achieved within the known formative time lag of $\sim 200 \mathrm{~ns}^{35}$ This approach is supported by the present observations which clearly illustrate that during a time interval of this magnitude, an avalanche chain or generation mechanism is active in the discharge buildup to the critical level. It should be noted, however, that when reference is made to electron avalanche growth initiated by a single electron, the expression $\exp \left[\int_{0}^{z_{11}} \bar{\alpha}(z) d z\right]$ does not represent the absolute magnitude of this growth since it is inherent in the definition of $\bar{\alpha}$ that the number of electrons participating in the process must everywhere be large; $\bar{\alpha}$ is a macroscopic parameter similar in concept to gas temperature and pressure. The integral, however, may be viewed as a relative measure of such growth in any given field.

The present experimental observations clearly indicate that a multiple avalanche process is operative involving photoionization of the gas. Such concepts have been assumed in the model of Nasser et al., ${ }^{4}$ which is a generalization of an earlier proposal by Loeb and Wijsman..$^{36}$ In this model, although gas photoionizing processes have been included and the subsequent accumulation of positive-ion space-charge effects considered, the apparent termination of the secondary photoelectric avalanches at the boundary of the primary avalanche head is not physically convincing (see Ref. 4, Fig. 2). A continued advance through the initial spacecharge volume must occur leading eventually to the displacement of $E_{\max }$ and the space-charge center along the axis of symmetry. In addition, there appears to be no physical reason to suggest that the presence of two spherical volumes of charge, tangentially in contact, can trigger the avalanche-to-corona transition by simply attaining charge equality. Moreover, as stated by Nasser, specific ionic distributions are in the final onset criterion not considered and the deciding factor is left purely to numbers. This does not sustain the physical claims attached to the model, although the physical approach up to this point reflects the observed sequence of events.
Thus, all of the existing methods for calculating coronaonset field strengths are of necessity empirical to a greater or lesser extent, and the onset criteria involved can include several parameters for which precise data are not known. However, owing to the dominating influence of the exponential term which is embodied in any breakdown criterion, the numerical predictions of the various models are seen not to differ significantly. ${ }^{4,32} \mathrm{~A}$ full appreciation of this aspect is to be gained from the comments of Zeleny ${ }^{37}$ and Thomson. ${ }^{38}$ Consequently, a simple approach is to be preferred; e.g., the criterion developed over 50 years ago by Schumann, ${ }^{2}$ particularly, as recent experimental studies ${ }^{39}$ indicate that the $\bar{\alpha}(E)$ data for atmospheric air can be expressed in the form originally deduced by that author. The application of this onset criterion to the electrode geometry used in the present study gives an onset field strength of $5.08 \mathrm{kV} / \mathrm{mm}$ compared with the measured value of $5.03 \mathrm{kV} / \mathrm{mm}$. The actual field distribution associated with the gap geometry was determined by a charge simulation method. ${ }^{40}$

In conclusion, it may be stated that the development of a rigorous physical-mathematical model of corona onset must await a full understanding of the avalanche-to-corona transition mechanism. Prior to this, little or nothing is to be gained in onset field strength calculations by increasing the model complexity.

'F.W. Peek, Jr., Dielectric Phenomena in High-Voltage Engineering, 3rd ed. (McGraw-Hill, New York, 1929).

${ }^{2}$ W.O. Schumann, Elektrische Durchbruchfeldstärke von Gasen (Springer, Berlin, 1923).

${ }^{3}$ A. Pedersen, IEEE Trans. Power Appar. Syst. PAS-86, 200 (1967).

${ }^{4}$ E. Nasser and M. Heiszler, J. Appl. Phys. 45, 3396 (1974).

${ }^{5}$ Les Renardières Group, Electra No. 23, 53 (1972).

${ }^{6}$ E. Bregnsbo, J.M. Christensen, G.C. Crichton, I.W. McAllister, and A.

Pedersen, IEE Conf. Publ. 118, 187 (1974).

'H. Norinder and O. Salka, Ark. Fys. 3, 347 (1951).

${ }^{8}$ E.N. Brago and I.S. Stekolnikov, Izv. Akad. Nauk SSSR, Otdel. Tekh.

Nauk 11, 50 (1958).

${ }^{9}$ E.M. Bazelyan, Zh. Tekh. Fiz. 34, 474 (1964).

${ }^{10}$ J.M. Meek and M.M.C. Collins, Electron. Lett. 1, 110 (1965).

${ }^{11} \mathrm{H}$. Raether, Electron Avalanches and Breakdown in Gases (Butterworths, London, 1964).

${ }^{12}$ I.W. McAllister, Proc. 13th International Conference on Phenomena in Ionized Gases, Berlin GDR, 1977, part I (VEB Buch-Export-Import, Leipzig, 1977), p. 365.

${ }^{13}$ M.R. Amin, J. Appl. Phys. 25, 210 (1954).

${ }^{14}$ H.A. Boyd, F.M. Bruce, and D.J. Tedford, Nature 210, 719 (1966).

${ }^{15}$ H. Schlumbohm, Z. Phys. 170, 233 (1962).

${ }^{16}$ J.H. Park and H.N. Cones, J. Res. Natl. Bur. Stand. 56, 201 (1956).

${ }^{17}$ G.A. Dawson and W.P. Winn, Z. Phys. 183, 159 (1965).

${ }^{18}$ I. Gallimberti, J. Phys. D 5, 2179 (1972).

${ }^{19}$ G.A. Dawson, J. Appl. Phys. 36, 3391 (1965).

${ }^{20}$ S.J. Dale, Ph.D. thesis, University of Strathclyde (1976).

"G. Pesavento, IEE Conf. Publ. 143, 253 (1976).

${ }^{22}$ E. Marode, J. Appl. Phys. 46, 2005 (1975).

${ }^{2}$ N. Ikuta and K. Kondo, IEE Conf. Publ. 143, 227 (1976).

${ }^{24}$ Les Renardières Group, Electra No. 53, 31 (1977).

${ }^{2}$ N. Ikuta, T. Ushita, and Y. Ishiguro, Elect. Eng. Jpn. 90, 52 (1970).

2"E. Marode, J. Appl. Phys. 46, 2016 (1975).

${ }^{27}$ A. von Engel, Ionized Gases, 2nd ed. (Oxford U.P., Oxford, 1965), p. 254.

${ }^{2}$ I.S. Stekolnikov, The Nature of the Long Spark (Izd. Akad. Nauk SSSR, Moscow, 1960), p. 57 
${ }^{29}$ G.G. Hudson and L.B. Loeb, Phys. Rev. 123, 29 (1961).

${ }^{30}$ H. Korge, K. Kudu, and M. Laan, in Ref. 12, p. 451.

"J.K. Hepworth, R.C. Klewe, and R.H. Varey, Electra No. 23, 107 (1972).

${ }^{12}$ M. Khaled, Elektrotechnische Z.-A 95, 369 (1974)

"S. Badaloni and I. Gallimberti, Electra No. 23, 107 (1972).

${ }^{i 4}$ H. Parekh, M.M.A. Salama, and K.D. Srivastava, J. Appl. Phys. 49, 107 (1978).
"M. Menes and L.H. Fisher, Phys. Rev. 94, 1 (1954).

${ }^{16}$ L.B. Loeb and R.A. Wijsman, J. Appl. Phys. 19, 797 (1948),

"J. Zeleny, J. Appl. Phys. 13, 444 (1942).

"J.J. Thomson and G.P. Thomson, Conduction of Electricity Through Gases 1I, 3rd ed. (Dover, New York, 1969), p. 520.

${ }^{34}$ H.A. Boyd and D.J. Tedford, J. Phys. D 4, 1140 (1971).

${ }^{4 !}$ H. Steinbigler, Elektrotechnische Z.-A 90, 663 (1969). 